\title{
CONSTITUTIVE LAWS FOR THIN PLATES OF POWER-LAW MATERIALS
}

\author{
C. H. Chol and J. PAN \\ Department of Mechanical Engineering and Applied Mechanics. The University of \\ Michigan. Ann Arbor. MI 48109. U.S.A. \\ and \\ S. C. TANG \\ Ford Motor Company, Dearborn. MI $4 \$ 12$ I. U.S.A.
}

(Recired 2 December 1989: in revised form 13 May 1990)

\begin{abstract}
Antract - With the Kirchhoff assumption. a stress resultant constitutive law as a function of the kinematic variables for thin plates of power-law hardening materials is derived under proportional straining conditions. Also, in analogy to the $J_{2}$ deformation plasticity and incremental plasticity theory. a How rule, based on the constitutive law, to describe the elastic plastic hehavior of the plate is proposed. The constitutive behavior of the plates subjected to uniaxial combined membrane force and bending moment is examined in detail and the results are compared with those for the corresponding clastic plastic materials using the through-lhe-thickness integration method. The yied surfices for puwer-law materials are conseructed and the formation of vertices on the yichat surfaces for perfectly plastic materials under such a loading condition is clearly demonstratted.
\end{abstract}

\section{INTROH)UCTION}

For the purpose of efficiently simulating shet metal forming processes by linite clement methods, plate or shell theories with the Kirchhofr assumption are usually adopted to reduce computational time. However, numerical integrations through the thickness of plates or shells under elastic plastic deformation are necessary. This increases the need of computational storage and time. Therefore, constitutive laws, where the stress resultants such as the membrane forces and moments are expressed as functions of the kinemalic variables such as the midplane strains and curvatures, are of interest.

The constitutive laws of incremental plasticity nature based on the stress resultants have been proposed by Crisfield (1974) and Bieniek and Funaro (1976) for thin plates of elastic-perfectly plastic materials as well as by Eggers and Kröplin (1978) and Eidsheim and Larsen (1981) for thin plates of elastic-plastic strain-hardening materials with a limit yield surface. Also, Morman et al. (1984) generalized these works to finite deformation formulation. Further applications of this type of constitutive law have been developed to analyze viscoplastic shells (Atkarsh et al., 1983), dynamically loaded plates (Lukkunaprasit and Kelly, 1979), stiflened plates (Kutt and Bienick, 1988), and composite laminated plates (Bank and Bienick. 1988).

We have employed the constitutive laws of Crisfield (1974), Bienick and Funaro (1976) and Eidsheim and Larsen (1981) to simulate sheet metal forming processes and found that the results of the computations agree well with the results using the throughthe-thickness integration method for elastic-perfectly plastic materials. However. for elastic-plastic strain-hardening materials, the computational results are not satisfactory when compared with the results using the through-the-thickness integration method. Consequently. we are interested in constructing these types of stress resultant constitutive laws for strain-hardening materials.

In a typical sheet metal forming process such as forming a body pancl of an automobile, the allowable highest plastic strains of material clements are about a few percent. The loading path of a material element may be nonproportional and complex. However. in order to construct a simple constitutive law in this paper. we make the assumption that all material elements experience nearly proportional loading conditions so that the use of the deformation plasticity theory is justified (Budiansky, 1959). Furthermore, since we are 


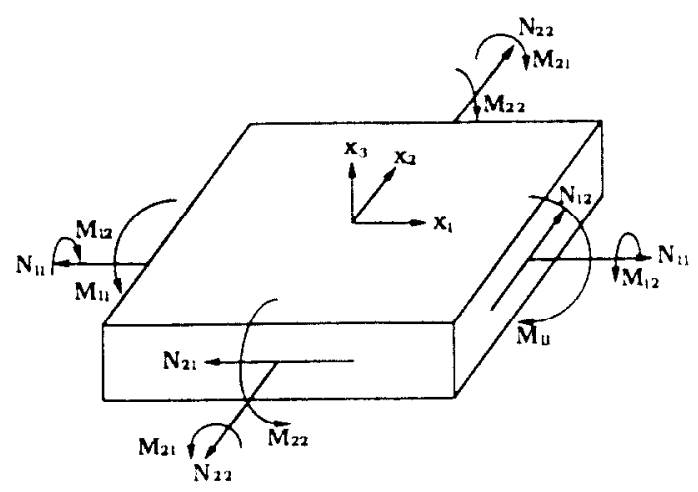

Fig. 1. The conventions of a plate element.

interested in the cases where the plastic deformation is large compared to the elastic deformation, the materials are assumed to be pure power-law materials. Then, the constitutive law can be expressed in terms of the stress resultants as functions of the currrent deformation state. In analogy to the work of Stören and Rice (1975), we can conceptually construct the yield surface and then formulate a flow rule of incremental plasticity nature in the stress resultant space to determine the work-conjugate strain rates as functions of the stress resultant rates.

In particular, the results based on the stress resultant constitutive laws for pure powerlaw malterials under uniaxial combined membrane fore and bending moment are compared with those for the corresponding elastic-plastic materials using the through-the-thickness integration method. The yield surfaces for the corresponding incremental plasticity theory are constructed. In the limit cases for power-law malterials, the yield surfaces for perfectly plastic materials are compared with those of Rice (1972) and Bienick and Funaro (1976).

\section{A STRESS RESULTANT CONSTITUTIVE LAW}

Within the context of the small-strain approath, the strains of at thin plate element, as shown in Fig. I, can be written with the Kirchhoff assumption as

$$
\varepsilon_{x \beta}=e_{x \beta}+\kappa_{x \beta} x_{1} \quad x, \beta=1,2
$$

where $e_{x / 1}$ represent the midplane strains and $k_{x / 1}$ represent the curvatures. In general, the plastic stratin as a function of the tensile stress of a tensile test for steels and aluminum alloys can be fitted by a power-law relation. We consider the calses where the plastic strain is much larger than the elastic strain. Therefore, we can use a pure power-law to describe the material stress-strain relation. The uniaxial tensile pure power-law stress-strain relation is shown in Fig. 2. The generalized multiaxial constitutive relation based on the $J_{2}$ deformation plasticity theory is

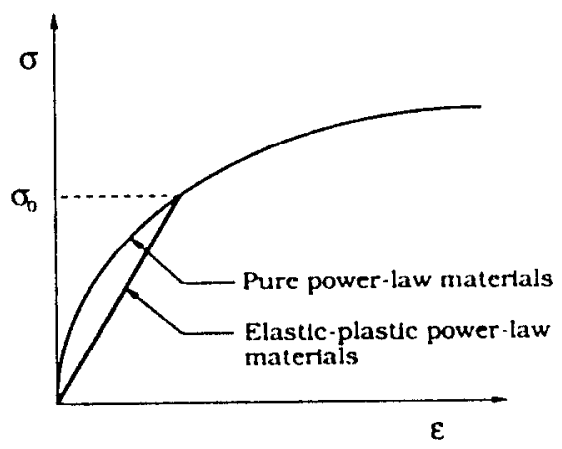

Fig. 2. The stress strain curves for pure power-law materials and the corresponding elastic plastic materials. 


$$
\varepsilon_{i j}=\frac{3}{2} \alpha\left(\frac{\sigma_{e}}{\sigma_{0}}\right)^{n-1} \frac{s_{i j}}{\sigma_{0}} \quad i, j, k=1,2.3,
$$

where $\varepsilon_{i j}$ are the strains, $\sigma_{e}\left(=\left[\frac{1}{3} s_{i j} s_{i j}\right]^{1 / 2}\right)$ the effective stress, $s_{i j}\left(=\sigma_{i j}-\frac{1}{3} \sigma_{k k} \delta_{i j}\right)$ the deviatoric stresses, $\boldsymbol{n}$ the hardening exponent, $\sigma_{0}$ the reference stress, and $x$ a material constant.

The stress field as a function of $x_{3}$ for the thin plate element shown in Fig. 1 can be derived by introducing eqn (1) into (2) with the condition of $\sigma_{33}=\sigma_{31}=\sigma_{32}=0$ :

$$
\sigma_{2 \beta}=Q\left(A_{x \beta}+B_{2 \beta} \cdot x_{3}\right)\left(D+E x_{3}+F x_{3}^{2}\right)^{(1-n), 2 n}, \quad x_{.} \beta, \gamma=1,2,
$$

where

$$
\begin{aligned}
Q & =\frac{2^{1 n} \sigma_{0}}{3^{(n+1)^{2 n}} x^{1, n},} \\
A_{x \beta} & =e_{i \beta} \delta_{x \beta}+e_{x \beta} . \\
B_{x \beta} & =\kappa_{x i} \delta_{x \beta}+\kappa_{x \beta} . \\
D & =e_{11}^{2}+e_{11} e_{22}+e_{22}^{2}+e_{12}^{2} . \\
E & =2 e_{11} \kappa_{11}+2 e_{22} \kappa_{22}+e_{11} \kappa_{22}+e_{22} \kappa_{11}+2 e_{12} \kappa_{12}, \\
F & =\kappa_{11}^{2}+\kappa_{11} \kappa_{22}+\kappa_{22}^{2}+\kappa_{i 2}^{2} .
\end{aligned}
$$

Then, the stress resultants $N_{\mathrm{x} / \mathrm{l}}$ and $M_{\mathrm{x} /}$ for the plate element can be derived as:

$$
N_{z / /}=\int_{-h / 2}^{h / 2} \sigma_{x \beta} \mathrm{d} x_{3}=Q \int_{-h / 2}^{h_{2}} \frac{A_{2 \mu}+B_{x / \mu} x_{3}}{\left(D+E x_{3}+F x_{3}^{2}\right)^{(n-1) / 2 n}} \mathrm{~d} x_{3}
$$

and

$$
M_{x \beta}=\int_{-h / 2}^{h, 2} \sigma_{x \mu /} \cdot x_{3} \mathrm{~d} x_{3}=Q \int_{-h / 2}^{h / 2} \frac{\left(A_{2 \beta}+B_{x \mu} \cdot x_{3}\right) \cdot x_{3}}{\left(D+E x_{3}+F x_{3}^{2}\right)^{(n-1) / 2 n}} \mathrm{~d} x_{3}
$$

where $N_{x / f}$ and $M_{x f}$ are the membrane forces and moments which are the functions of $e_{x f}$. $k_{x / \beta}$ and $n$. These functions are independent of $x_{3}$ since we carry out the integrations over a finite interval of $x_{3}$. Now, if we examine eqns (4) and (5) for nonlinear elastic power-law materials, the constitutive law relating the stress resultants $N_{x / f}$ and $M_{x \beta}$ to the kinematic variables $e_{x p}$ and $\kappa_{x p}$ is completely specified.

Assuming all the material through the thickness is subject to the same proportional straining condition, we have

$$
\frac{\varepsilon_{22}}{\varepsilon_{11}}=\frac{e_{22}\left(1+\frac{\kappa_{22}}{e_{22}} x_{3}\right)}{e_{11}\left(1+\frac{\kappa_{11}}{e_{11}} x_{3}\right)}=C_{1}
$$

and

$$
\frac{\varepsilon_{12}}{\varepsilon_{11}}=\frac{e_{12}\left(1+\frac{\kappa_{12}}{e_{12}} x_{3}\right)}{e_{11}\left(1+\frac{\kappa_{11}}{e_{11}} x_{3}\right)}=C_{2},
$$

where $C_{1}$ and $C_{2}$ are constants. Because 
and

$$
\frac{\sigma_{22}}{\sigma_{11}}=\frac{2 \frac{\varepsilon_{32}}{\varepsilon_{11}}+1}{2+\frac{\varepsilon_{22}}{\varepsilon_{11}}}=\frac{2 C_{1}+1}{2+C_{1}}=C_{3}
$$

$$
\frac{\sigma_{12}}{\sigma_{11}}=\frac{\frac{\varepsilon_{12}}{\varepsilon_{11}}}{2+\frac{\varepsilon_{22}}{\varepsilon_{11}}}=\frac{C_{2}}{2+C_{1}}=C_{4}
$$

where $C_{3}$ and $C_{4}$ are constants. a proportional straining condition is equivalent to a proportional stressing condition. At any $x_{3}$, the necessary and sufficient conditions to satisfy equs (6) and (7) are

and

$$
\begin{aligned}
& \frac{c_{22}}{e_{11}}=C_{1}, \\
& k_{22}=\frac{c_{22}}{c_{11}},
\end{aligned}
$$

$$
\begin{aligned}
& c_{12}=c_{2} \\
& c_{11} \\
& k_{12}=c_{12} . \\
& k_{11}=
\end{aligned}
$$

With equs (8) and (9), eqn (3) can be simplified as

$$
\sigma_{x / s}=A_{x \beta} Q R\left|1+\frac{k_{11}}{c_{11}} x_{3}\right|^{1, n} \operatorname{sign}\left(1+\frac{k_{11}}{e_{11}} x_{3}\right)
$$

where

$$
R=\left(e_{11}^{2}+e_{11} e_{22}+e_{22}^{2}+e_{12}^{2}\right)^{\left(1-n n_{1} 2 n\right.}
$$

The function sign ( ) equals 1 when its argument is positive and equals -1 when its argument is negative. Hence, the stress resultants are derived as follows:

$$
\begin{aligned}
N_{x / S} & =A_{x \beta} Q R \int_{n, 2}^{h^{\prime} 2}\left|1+\frac{k_{11}}{e_{11}} x_{3}\right|^{1 n} \operatorname{sign}\left(1+\frac{k_{11}}{e_{11}} x_{3}\right) \mathrm{d} x_{3} \\
& =A_{x / 3} \frac{c_{11}}{k_{11}} \frac{n}{n+1} Q R\left[\left|1+\frac{k_{11} h}{2 e_{11}}\right|^{(n+1) \cdot n}-\left|1-\frac{k_{11} h}{2 e_{11}}\right|^{(n+1) n}\right]
\end{aligned}
$$

and

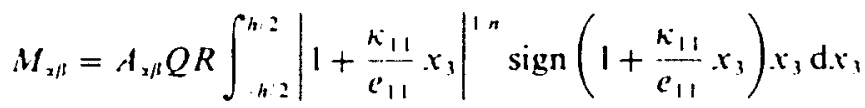

$$
\begin{aligned}
& =A_{x \beta} e_{k_{i 1}^{2}}^{i} \frac{n}{2 n+1} Q R\left[\left|1+\frac{\kappa_{11} h}{2 e_{11}}\right|^{(2 n+1) n} \operatorname{sign}\left(1+\frac{k_{11} h}{2 e_{11}}\right)\right. \\
& \left.-\left|1-\frac{\kappa_{11} h}{2 e_{11}}\right|^{12 n+11 n} \operatorname{sign}\left(1-\frac{\kappa_{11} h}{2 e_{11}}\right)\right]-\frac{e_{11}}{\kappa_{11}} N_{x \beta} \text {. }
\end{aligned}
$$


Note that eqns (11) and (12) involve the ratio $\kappa_{1 /} / e_{11}$. We can define a ratio $C$ such that

$$
2 C=\kappa_{1}, h_{i} e_{11}
$$

Then eqns (11) and (12) can be expressed as

$$
N_{x \beta}=Q R A_{x \beta} h f(C . n)
$$

and

$$
M_{x \beta}=Q R A_{x \beta} h^{2} g(C \cdot n)
$$

where $f$ and $g$ are functions of $C$ and $n$ only. The amplitudes of $N_{x \beta}$ and $M_{x \beta}$ are now related to that of the strain by $R A_{z f f}$ which represents a quantity with the amplitude of the strain to $l / n$ power. In this way, these two equations are in agreement with the general results for power-law materials given as eqns (8) and (9) in Chou et al. (1988).

In pure bending cases, the midplane strains are equal to zero under the Kirchhoff assumption. Equation (3) can be reduced to

$$
\sigma_{x, \beta}=B_{x / \beta} Q F^{(1-n) 2 n}\left|x_{3}\right|^{1 ; n} \operatorname{sign}\left(x_{y}\right)
$$

Hence, the stress resultants are

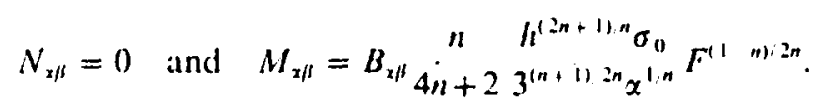

As $n$ approaches infinity in eyns (11) and (12) for perfectly plastic materials, the stress resultants caln be expressed as

$$
N_{x l}=A_{x \beta} \frac{e_{11}}{k_{11}} \frac{\sigma_{01}}{\sqrt{3 D}}\left(\left|1+\frac{\kappa_{11} h}{2 e_{11}}\right|-\left|1-\frac{\kappa_{11} h}{2 e_{11}}\right|\right)
$$

and

$$
M_{x / h}=A_{x h} \frac{e_{i 1}^{2}}{k_{11}^{2}} \frac{\sigma_{01}}{2 \sqrt{3 D}}\left[\left(1+\frac{\kappa_{11} h}{2 e_{11}}\right)\left|1-\frac{\kappa_{11} h}{2 e_{11}}\right|-\left(1-\frac{k_{11} h}{2 e_{11}}\right)\left|1+\frac{k_{11} h}{2 e_{11}}\right|\right] .
$$

\section{UNIAXIAL COMBINED LOADINGS}

We consider a thin plate subjected to uniaxial combined membrane force $N_{11}$ and bending moment $M_{1}$, with the plane strain condition $\left(\varepsilon_{3 i}=0, i=1,2,3\right)$ and with the plane stress (simple beam) condition $\left(\sigma_{2 i}=0, i=1,2,3\right)$. From eqns (11) and (12), we have

$$
N_{11}=\Gamma \frac{n}{n+1} \frac{\sigma_{0}}{\alpha^{1 n}} \kappa_{11}{ }^{\prime}\left\{\left|e_{11}+\frac{\kappa_{11} h}{2}\right|^{(n+1) \cdot n}-\left|e_{11}-\frac{\kappa_{11} h}{2}\right|^{(n+1) n}\right\}
$$




$$
\begin{aligned}
& M_{11}=\Gamma \begin{array}{cc}
n & \sigma_{0} \\
2 n+1 & x^{1 n} \kappa_{11}
\end{array}\left[\left|e_{11}+\frac{\kappa_{11} h^{112 n+11 n}}{2}\right|^{\operatorname{sign}}\left(e_{11}+\frac{\kappa_{11} h}{2}\right)\right.
\end{aligned}
$$

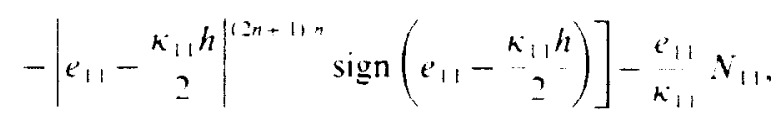

where $\Gamma=1$ for the plane stress condition and $\Gamma=(2 \sqrt{3})^{n+1 n}$ for the plane strain condition.

To check the validity of eqns (18) and (19), we compare the constitutive behavior with that for the corresponding elastic-plastic materials using the through-the-thickness integration method. The stress-strain curves for pure power-law materials and the corresponding elastic-plastic materials are shown in Fig. 2. For the convenience of presentation of the results under combined membrane force and bending moment, we denote $N$ for $N_{11}$. $M$ for $M_{11}$. $e$ for $e_{11}$, and $k$ for $\kappa_{11}$. Also, we normalize the results of $N$ by $N_{L}\left(=\sigma_{11} h\right), M$ by $M_{L}\left(=\sigma_{0} / h^{2} / 4\right)$. $e$ by $e_{L}\left(=\sigma_{0} / E\right)$, and $k$ by $\kappa_{L}\left(=3 \sigma_{0} / E h\right)$. In Figs $3-6$ where the moment-curvature relations are plotted. P-curves (solid lines) represent the results of eqn (19) and E-P curves (dotted lines) represent the results for the corresponding elastic-plastic materials using the through-the-thickness integration method.

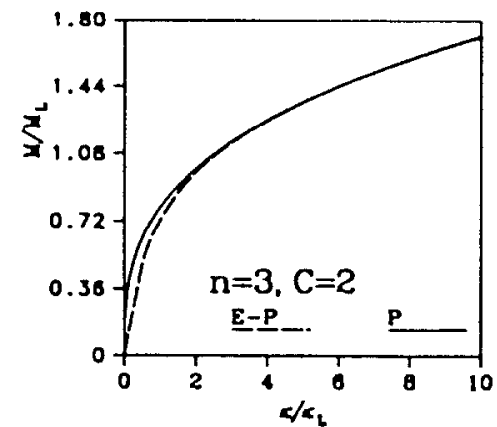

(a)

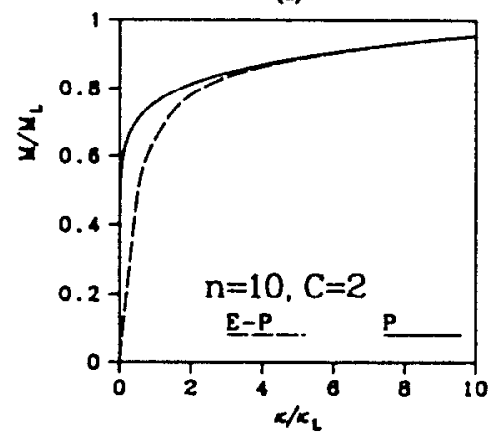

(b)

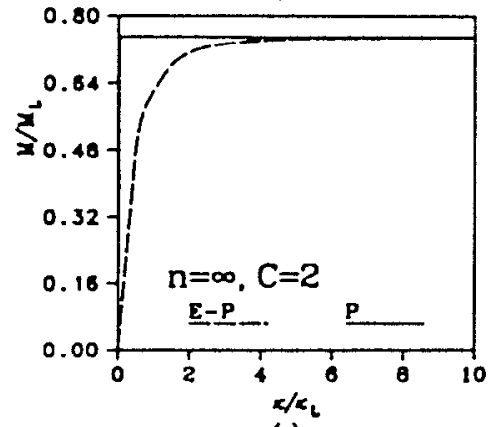

(c)

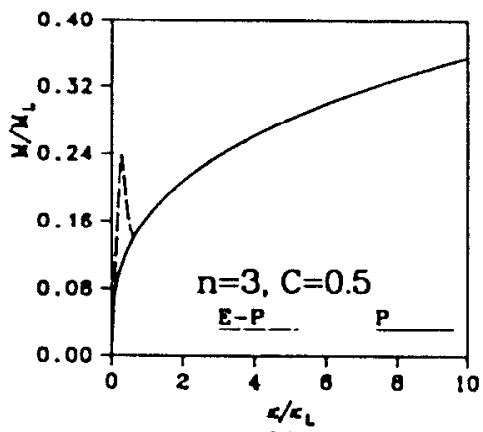

(d)

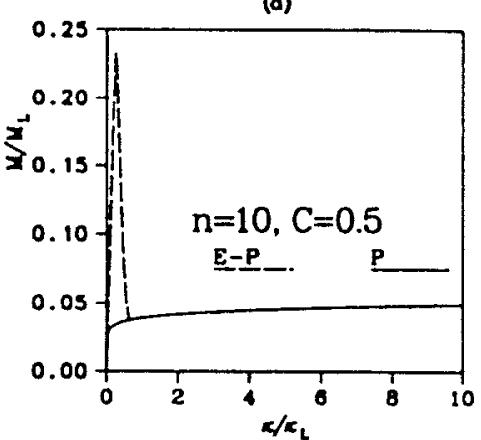

(c)

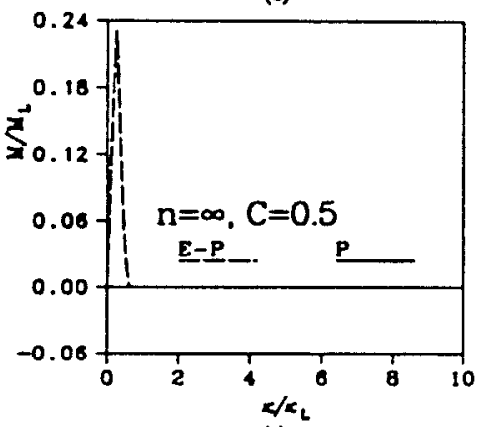

(n)

Fig. 3. Comparisons of the plane stress moment curvature results under proportional stretching and bending for pure power-faw materials and the corresponding chastic plastic materials. 


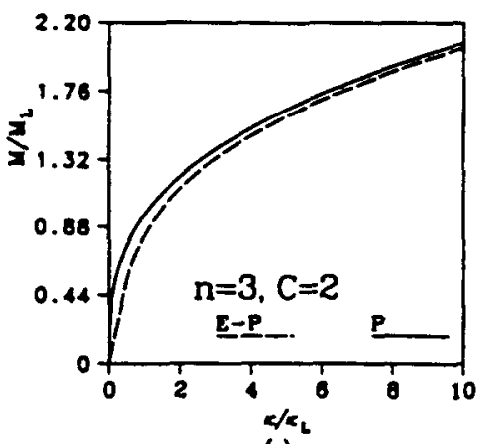

(a)

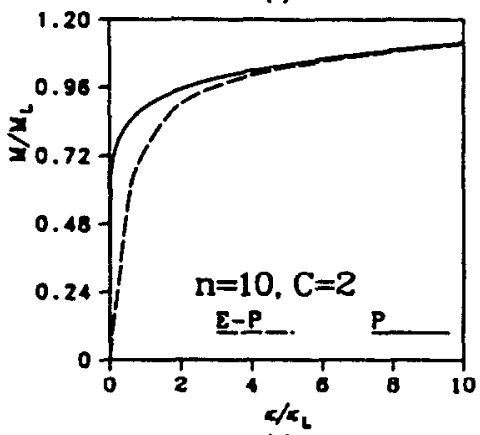

(b)

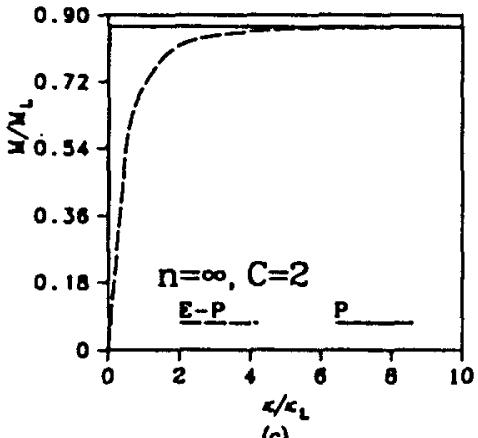

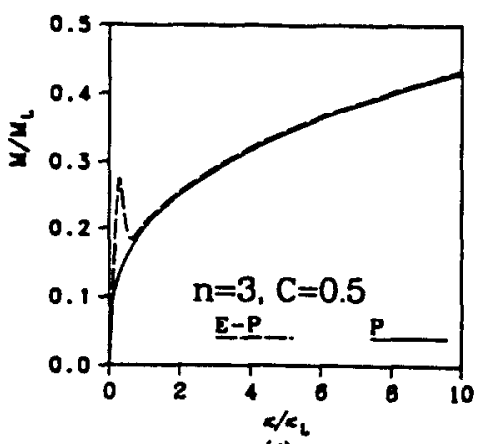

(d)

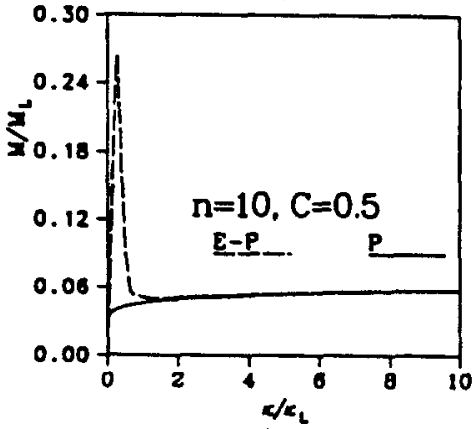

(6)

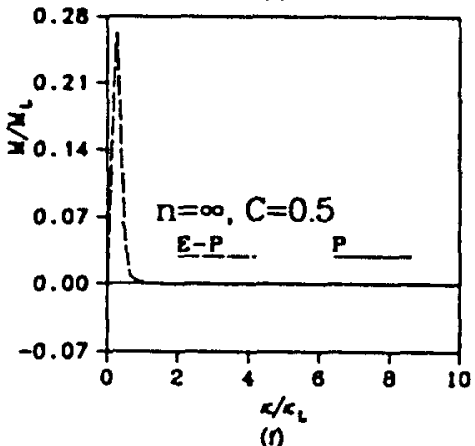

Fig. 4 . Comparisons of the plane strain moment curvalture results under proportional stretching and bending for pure power-law matterials and the corresponcting elastic plastic materials.

First, we examine the cases where ratio $C(=h / h 2 c)$ as defined in eqn $(13)$ is fixed during the monotonically increasing proportional straining processes. Figure 3 shows the normalized moment-curvature relationship under platle stress conditions for $C=0.5$ and 2 and $n=3,10$, and $s$. In the ligures. P-curves and E-P curves become close to each other when $\kappa / \kappa_{l}$, becomes large. For small hardening exponent 11 , as shown in Fig. 3a.d, the results based on eqn (19) give a very good approximation compared to the through-the-thickness integration results. When $C^{\prime}<1$, as shown in Fig. $3 \mathrm{~d}$ - $f^{\circ}$ each of the curves for elastic-plastic materials hats a peak becaluse the elastic zone dominates the distribution of the moment initially.

Figure 4 presents the normalized moment-curvature results under plane strain conditions for $C=0.5$ and 2 and $n=3,10$, and 5 . We can see in the figures that the general trends are the same as those under plane stress conditions except that when $k$ becomes large. both curves do not inatch exactly in each calse. The reason is that Poisson's ratio alfects the results in the elastic zone under plane strain conditions. Here, we use $v=0.3$ for the corresponding elastic-plastic materials whereas the pure power-law malterials modeled by the $J_{2}$ deformation plasticity theory are incompressible. If we increase the value of Poisson's ratio for the corresponding elastic-plastic materials, the difference of both curves in each case will decrease. In general, the pure power-law results agree well with the elastic-plastic through-the-thickness integration results when $\kappa / \kappa_{t}>5$. Note that $\sigma_{0} / E$ is 

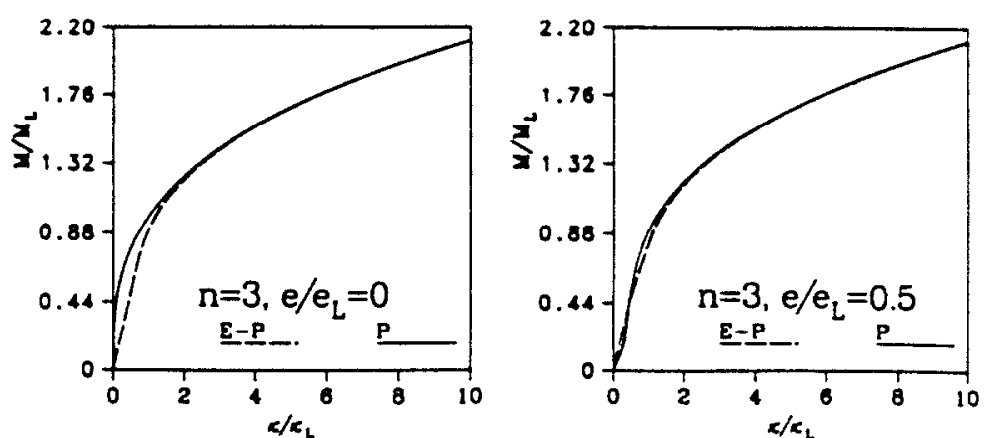

(a)

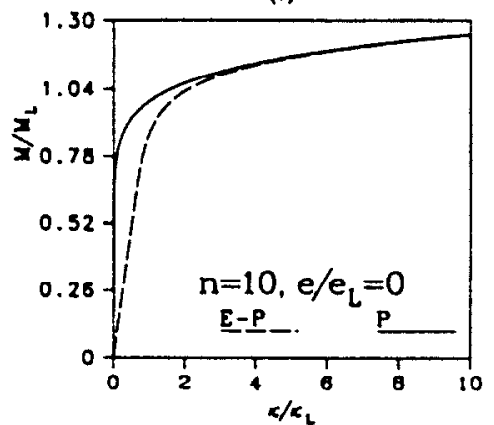

(d)

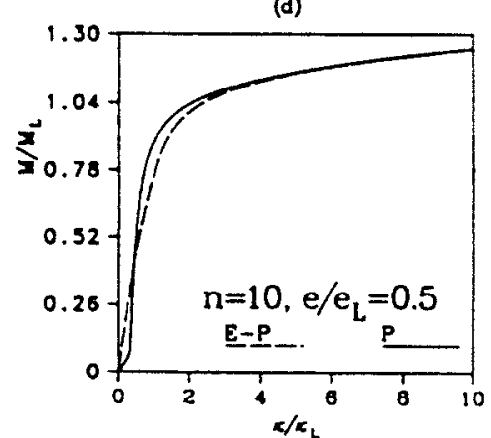

(b)

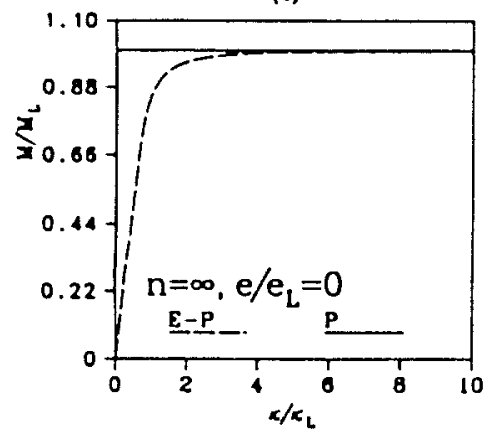

(c)

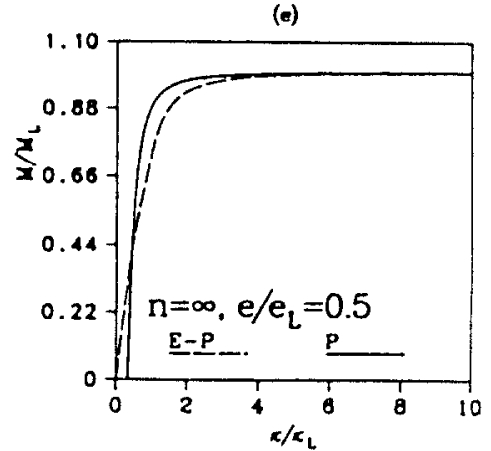

(I)

Fig. 5. Comparisons of the plane stress moment curvature results subjected to constant midplane strain for pure power-law materials and the corresponding clastic plastic materials.

of the order of $10^{-3}$ for structural steels and aluminum alloys. Therefore, when the strain is larger than 1\%, the pure power-law results will give good approximations for the elastic-plastic behavior of the plates.

Figures 5 and 6 are the results of the normalized moment-curvature relationship for plane stress cases and plane strain cases under pre-strained loading conditions, where a small midplane strain is kept constant while the curvature increases. Figure 5 a $c$ shows the plane stress results under pure bending conditions for $n=3,10$, and $x$, respectively. Figure $5 \mathrm{~d}-f$ shows the plane stress results under the pre-strain condition of $e^{\prime} e_{t}=0.5$ for $n=3$. 10 , and $x$, respectively. Figure $6 a-c$ shows the plane strain results under pure bending conditions for $n=3,10$, and $\propto$, respectively. Figure 6d -f shows the plane strain results under the pre-strain condition of $e / e_{L}=0.5$ for $n=3,10$, and $x$. respectively. In each of these figures, in spite of some differences at the initial bending stages, both curves become close to each other at large $\kappa / \kappa_{t}$. However, as in the monotonically increasing proportional straining cases. Fig. 6a-f shows there exist slight differences between the pure power-law results and those for the corresponding elastic-plastic materials under plane strain conditions as $k / k$, becomes large. This is due to the Poisson's ratio effect as explained carlier. As shown in Fig. $5 \mathrm{~d}-\mathrm{f}$ for the plane stress cases and in Fig. $6 \mathrm{~d}-\mathrm{f}$ for the plane strain cases. there exist transition points with discontinuous slopes for pure power-law materials. This is due to sign $\left(e_{11}-\kappa_{1}, h / 2\right)$ in eqn (19). from which different functional forms of the solutions are generated for $C$ larger and less than unity. 

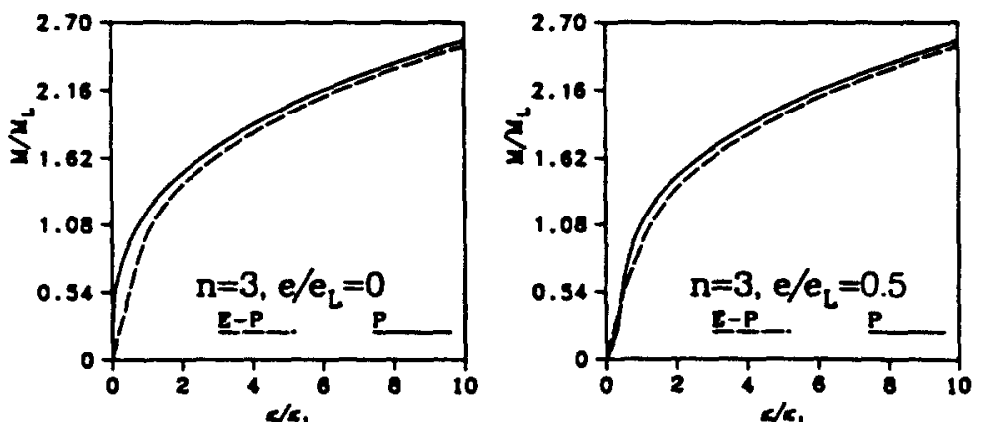

(a)

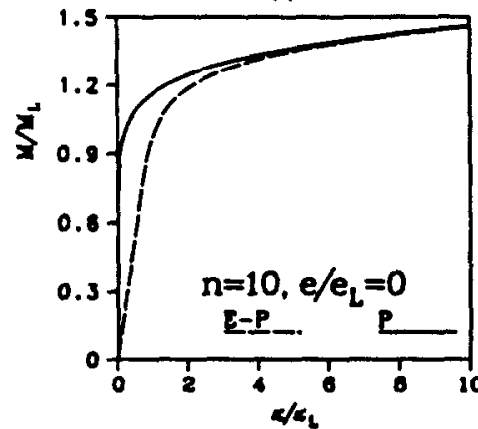

(d)

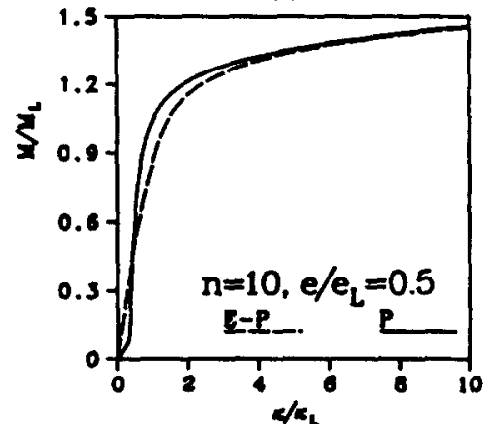

(b)

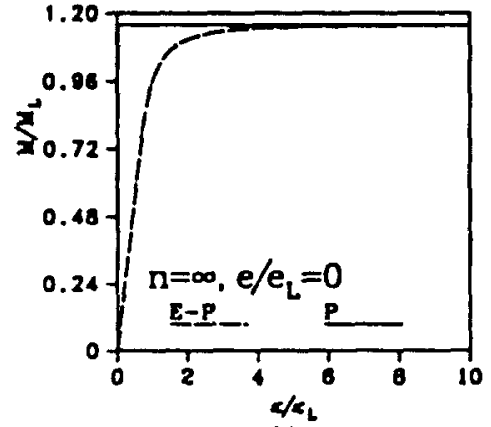

(1)

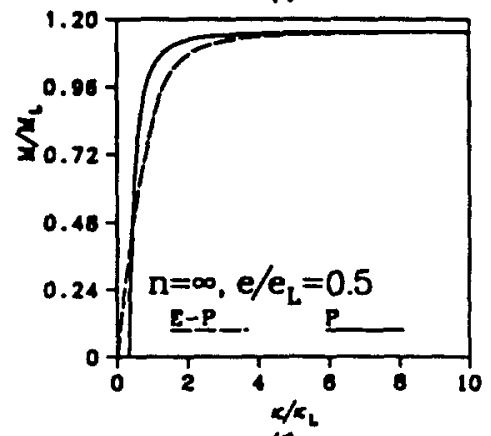

(n)

Fig. 6. Comparisons of the plane strain moment curvature results subjected to constant midplane strain for pure power-law malterials and the corresponding elastic-plastic materials.

\section{COMPLEMENTARY POTENTIAL SURFACES}

To facilitate further discussions, we define the stress resultant vector $\mathbf{S}$ and the generalized strain vector e as

$$
\mathrm{S}=\left\{N_{11}, N_{22}, N_{12}, M_{11}, M_{22}, M_{12}\right\}
$$

and

$$
\mathbf{e}=\left\{e_{11}, c_{22}, 2 e_{12}, k_{11}, k_{22}, 2 k_{12}\right\}
$$

As shown in eqns (11) and (12), the constitutive law between $S$ and $\mathbf{e}$ is completely specified under proportional straining conditions. When we construct the finite element formulation by variational principles, the tangent modulus $\mathbf{D}(\dot{S}=\mathbf{D} \dot{\mathbf{e}})$ for numerical iterations is needed and it can be derived easily. But the approach mentioned above is valid only for power-law nonlinear elastic materials.

For elastic-plastic materials, phenomenological plasticity theories based on the invariants of $N_{x \beta}$ and $M_{x \beta}$ and interpolation between the initial yield surface and the limit yield surface are available in Crisfield (1974). Bieniek and Funaro (1976), and Eidsheim and Larsen (1981). In Eidsheim and Larsen (1981), material plastic hardening is incorporated within the framework of Crisfield (1974) and Bieniek and Funaro (1976) with the 


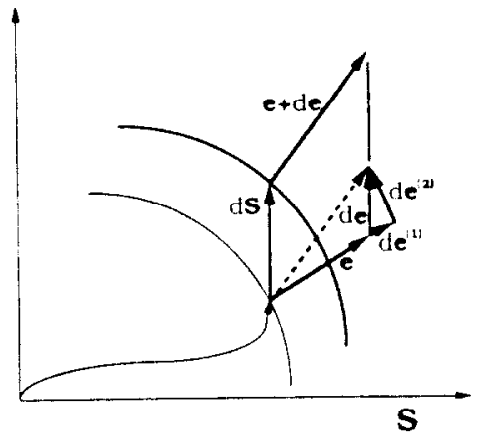

Fig. 7. The stress resultant increment ds and the effective generalized strain increment de' in the stressubuhant space.

assumption that the effective stress will asymptotically reach a limit value and therefore the limit yield surface is still applicable. However, the results are not satisfactory when these constitutive laws were used in simulating the forming processes of thin sheets of plastic hardening matterials. Therefore, we are interested in constructing a phenomenological plasticity theory where material plastic hardening can be accounted for.

Of course, the constitutive law of the form given by equs (11) and (12) is applicatble when the plastic delomation is large compared with the elastic deformation and the loading path is nearly proportional. Ilowever, during sheet metal forming processes. clastic unloading may occur and the yied conditions are theretore needed to determine the state of elastic unloading or plastic loading after initial unloadling

Motivated by the construction of the rate form of the $J$. deformation plasticity in Stören and Rice (1975), we can construct dec flow rule for the corresponding incremental plasticity theory based on the deformaltion plasticity relation of $S$ and $e$ in cyns (II) and (12). As shown in Stören and Rice (1975), when we seck the rate form of the constitutive law for Mises matcrials, the plastic strain rate in the deformation platstictity theory uan be decomposed into two components. One can be identilied ats the component given by the constitutive latw of the corresponding incremental plasticity for Mises matlerials. The other is at at tangent to the Mises yield surfate. As sketched in fig. 7. dS and de of a pure powerlaw deformation plasticity material alle shown at a given $S$ and e in the $S$ spatce. We

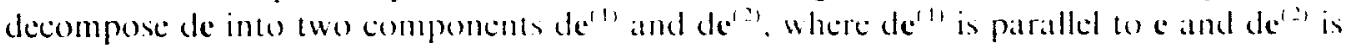
normal to e. Under proportional loading conditions. de is aro and de" equals de: the yicld surface. by delintion with the atssumption of normality. must be normal to de or e. Therefore. we catn construct the yiedd surface of incremental plasticity, which is normal to e, in the $S$ space based on the constilutive law of eqns (II) and (12). In this way, the plastic response ol incremental plasticity concides with that of deformation plasticity under proportional loading conditions.

The yietd surface constructed in this way really corresponds to the complementary potential surface of detormation plasticity. The complementary potential if of deformation plasticity is defined ats

$$
c_{i}=\frac{i \mu}{i S_{i}}
$$

which indicates that the generalized strain vector is normal to the complementary potential surface. For a constant complementary potential ' $y$ ' in the $S$ space. We have

$$
\mathrm{dW}=\underset{i S_{i}}{i S_{i}}=c_{1}, \mathrm{~d} S_{1}=0
$$

For the purpose of illustration. we will construct the complementary potential surfaces 
(or the yield surfaces of the corresponding incremental plasticity theory) under uniaxial combined membrane force $N$ and bending moment $M$ in the following:

$$
\mathrm{d} \Psi=0=e \mathrm{~d} N+\kappa \mathrm{d} M \text { or } \frac{\mathrm{d}(M / h)}{\mathrm{d} N}=-\frac{1}{2 C} .
$$

where $C$ is defined in eqn (13). According to eqns (18) and (19), the ratio of $M / h$ to $N$ is a function of $C$ only. That is.

$$
\frac{M / h}{N}=F(C) \text { or } C=F^{-1}\left(\frac{M / h}{N}\right)
$$

where $F^{-1}$ represents the inverse function of $F$. To find the yield surface, we can integrate eqn (24) starting from an arbitrary set $\left(N^{0}, M^{0}\right)$. Note that for pure power-law materials, the yield surfaces (or the complementary potential surfaces) are self-similar. Here we choose $V^{\prime \prime}=x \sigma_{10} h$ and $M^{\prime \prime}=0$, where $x=1$ in plane stress cases and $x=2 / \sqrt{3}$ in the plane strain cases. The function of the yield surface is represented by an integral equation as

$$
\frac{M}{h}=\int_{v^{\prime \prime}}^{v}-\frac{1}{2 F^{-1}\left(\frac{M / h}{N^{\prime}}\right)} \mathrm{d} N^{\prime} .
$$

We can numerically integrate eyn (25) and plot $\bar{M}\left(=M / M_{t}\right)$ as a function of $\bar{N}\left(=N / N_{t}\right)$ for several values of $n$.

Figure 8a,b shows the yield surfaces under plane stress conditions and under plane strain conditions, respectively, for $n=1,3,7$, and $\infty$. The surfaces under plane stress conditions, ats shown in Fig. 8a, are smaller than those under plane strain conditions, ats shown in Fig. $8 \mathrm{~b}$, for each $n$ beciuse there are more constraints in the latter calse. Due to the factors $\left|e_{11}+k_{1}, h / 2\right|$ and $\left|e_{11}-k_{1} h / 2\right|$ in eyns (18) and (19), there are four sets of constitutive equations depending on $C$. Thus, in the stress resultant space, there are four
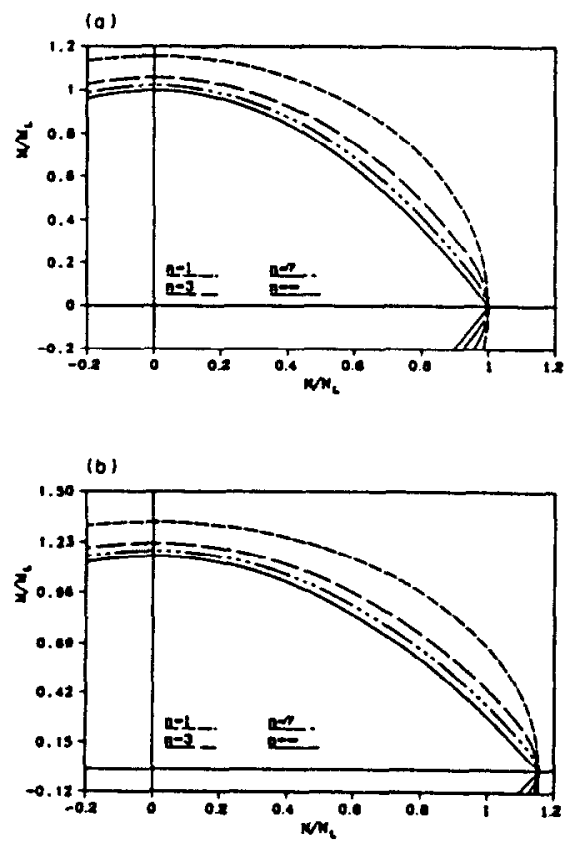

Fig. 8. The yield surfaces in the $\bar{M}-\bar{N}$ plane under plane stress conditions (a) and plane strain conditions (b). 


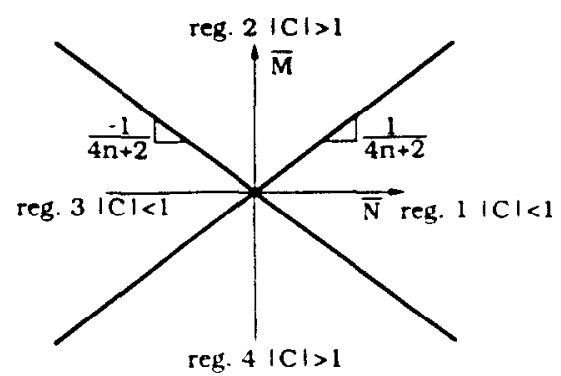

Fig. 9. Four subdomains divided by two transition lines in the $\overline{\bar{H}}-\overline{\mathrm{N}}$ plane

subdomains corresponding to these four sets of constitutive equations. These four subdomains are separated by two transition lines $(C= \pm 1)$ which pass through the origin with the slopes $\pm(4 n+2)^{-1}$, as shown in Fig. 9. The slopes of the yield surfaces are continuous when the yield surfaces pass through the transition lines. In Fig. 8a.b, when $n$ increases, the curvatures of the yield surfaces at the $\bar{N}$ axis increase. Also. the magnitude of the slopes of the transition lines decreases such that the size of subdomains 1 and 3 decreases. Consequently, when $n$ appro:sches infinity, the slopes of the transition lines become zero and subdomains 1 and 3 degenerate and coincide with the $\bar{N}$ axis. Therefore. only subdomains 2 and 4 exist for $n=\infty$. Because the yield surface goes through subdomain 2 into subdomain 4 directly, it is clearly shown that the slopes are discontinuous at $\bar{M}=0$ and so the vertices are formed, as shown in Fig. 8a,b for $n=\infty$.

Figure 10 shows the comparison between the yield surface of perfectly plastic material under plane stress conditions and the limit yield surface of Bienick and Funaro (1976). Both surfaces are very close to each other. Our yield surface has a continuous slope at $\bar{N}=0$, but the yield surface of Bienick and Funaro (1976) does not. Under plane strain conditions, the yicld surface for perfectly plastic materials $(n=\infty)$, as shown in Fig. 8 b. is the same as the parabolic yield surfice of Rice (1972).

We curve-fit the yield surface by second order and fourth order polynomials for future application under plane stress conditions. Table I lists the constants of these equations. We can obtain good fits by second order polynomials for low hardening matterials (with $n>3$ ). For high hatrdening materials (with $n<3$ ), fourth order polynomials are needed to give good fits.

Following the same procedure, we can derive the equation for the yield surfaces under pure biaxial bending and twisting conditions from eqn (15). The result for any hardening power $n$ is

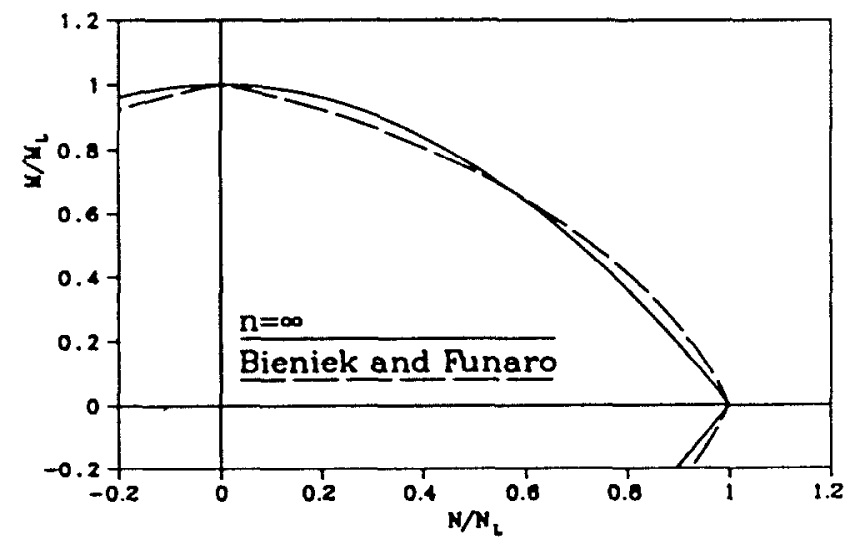

Fig. 10. Comparisons of the yield surface for $n=\propto$ for pure power-law materials with the limit yield surface of Bieniek and Funaro (1976). 
Table 1

\begin{tabular}{|l|r|r|r|r|r|r|r|r|r|r|}
\hline $\mathrm{n}$ & $\mathrm{A}$ & $\mathrm{B}$ & $\mathrm{C}$ & $\mathrm{D}$ & $\mathrm{E}$ & $\mathrm{F}$ & $\mathrm{G}$ & $\mathrm{H}$ & $\mathrm{J}$ & $\mathrm{K}$ \\
\hline 1 & -6 & 1 & -0.77 & 1.16 & -35 & -5 & 1 & -0.24 & -0.59 & 1.16 \\
\hline 3 & -16 & 1 & -0.93 & 1.06 & 3846 & -33 & 1 & -0.11 & -0.83 & 1.06 \\
\hline 5 & -26 & 1 & -0.96 & 1.04 & 1077 & -28 & 1 & -0.05 & -0.92 & 1.04 \\
\hline 7 & -35 & 1 & -0.97 & 1.03 & 33040 & -71 & 1 & -0.03 & -0.94 & 1.03 \\
\hline$\infty$ & & & -1 & 1 & & & & 0.004 & -1.004 & 1 \\
\hline
\end{tabular}

$\begin{array}{lll}\text { Region 1: } \bar{N}=A \bar{M}^{2}+B & \text { or } & \bar{N}=E \bar{M}^{4}+F \bar{M}^{2}+G \\ \text { Region 2: } \bar{M}=C \bar{N}^{3}+D & \text { or } & \bar{M}=H \bar{N}^{4}+J \bar{N}^{2}+K\end{array}$

$$
M_{11}^{2}-M_{11} M_{22}+M_{22}^{2}+3 M_{i 2}^{2}=q \text {. }
$$

where $q$ is a function of the hardening exponent $n$ and the kinematic variables. In other cases, we can obtain the yield surfaces using the numerical integration method.

\section{DISCUSSION}

In incremental plasticity theories, the flow rule is needed to establish the rate form of the constitutive relation. Here, we propose a flow rule based on the nonlinear elastic (deformation plasticity) behavior of eqns (3). (11), and (12) in analogy to the work of Stören and Rice (1975). The rate form of the incremental plasticity is expressed as

$$
S=D^{(1)} \cdot \dot{\mathbf{i}}^{(1)},
$$

where $D^{(1)}$ represents the tangent modulus and $\dot{c}^{(1)}$ is the effective generalized strain ratte. Note that the rate form of the deformation plasticity theory is

$$
S=D \cdot \dot{\mathbf{c}}
$$

where $D$ can be derived from eqns (11) and (12). Referring to Fig. 7. the effective generalized strain rate in the corresponding incremental plasticity theory is

$$
\dot{\mathbf{e}}^{(1)}=\mathbf{n}(\mathbf{n} \cdot \dot{\mathbf{e}}) \text {, }
$$

where $\mathbf{n}=\mathbf{c} /|\mathbf{c}|$. Substitution of eqn (28) into eqn (29) yiclds

$$
\dot{\mathbf{e}}^{(1)}=\mathbf{n}\left(\mathbf{n}^{\mathrm{r}} \cdot \mathbf{D}^{-1} \cdot \dot{\mathbf{S}}\right) \text {. }
$$

where D 'represents the inverse of D. From eqne (27) and (30), the tangent modulus $D^{\prime 11}$ of the corresponding incremental plasticity theory can be expressed ats

$$
D^{(1)}=\left[n\left(n^{r} \cdot D^{-1}\right)\right]^{-1} \text {. }
$$

For determining the loading or unloading condition easily, we define $\xi=S \cdot n$. Plastic deformation occurs when $\xi>0$. Otherwise, there is only elastic deformation and the usual elastic modulus for elastic thin plates will be used. In addition, an initial yicld surface must be defined for specifying the elastic domain in the stress resultant space. However, we are interested in the cases where the plastic deformation is much larger than the elastic deformation. Therefore, a reasonable choice of the initial yield surface may not affect the global results significantly. The proposed flow rule, of course, is restricted to nearly 
proportional loading conditions. Further numerical experiments are needed to examine the applicability of the proposed flow rule.

tikmuledgement - The authors acknowledge the support of this work by the Unwersity Research Program of Ford Motor Company.

\section{REFERENCES}

Atkatsh. R. S. Bieniek. M. P. and Sandler, I. S. (1983). Theory of viscoplastic shells for dynamic response. $J$ Appl. Me'ch. Trans. ASIME 50, 131-136.

Bank. L. C. and Bieniek. M. P. (1988). Stress-resultant plasticity theories for composite laminated plates. Int. J. Plaxticity 4. 317-333.

Bieniek. M. P. and Funaro. J. R. (1976). Elasto-plastic behavior of plates and shells. Report No. DNA 3954T, Weidlinger Associates. New York.

Budiansky. B. (1959). A reassessment of deformation theories of plasticity. J. Appl. Mech. Trans. ASIIE 26, $259 \cdot 264$.

Chou. C. H.. Pan. J. and Tang. S. C. (1988). Elastic plastic constitutive laws for thin plates. Enqinecring Science Preprint, ESP25 88023. 25th Annual Technical Meeting. Soctety of Engmeering Science. Berkeley, CA. 2022 June.

Crisficld. M. A. (1974). On an approximate yicld criterion for thin stecl shells. TRRL Report LR658. Transport and Roatd Rescarch Laboratory. Crowthorne, Berkshire.

Eggers. H. and Kröplin. B. (1978). Yielding of plates with hardening and large deformations. Int. J. Numer. lfeth. Enang 12.739 750.

Firdsheim. O. M and Larsen. P. K. (1981). A study of some generalized constitutive methods for elasto-plastic shells. In Vomlinear Finite Element Analysix in Structural Mechanics (Edited by W. Underlich, K. J. Bathe and E. Stein). pp. 364 - 384. Springer. Berlin

Kutt, L. M. and Bienick. M. P. (1988). Elasto-plastic constitutive cquations of stiflened plates. J Engme Mech 114.656070 .

Lukkunaprasit. P. and Kelly, J. M. (1979). Dynamic plastic analysis using stress resultant finite element formulation. Int. J. Solids Sirurcures 15, 211 - 240.

Morman. K. N.. Jr., Tang. S. C. and Mathers, M. D. (1984). Intrinsic constitutive equations for the targe deformation analysis of clastic plastic thin shells. Tiechnical Report SR-84-107, Ford Motor Company.

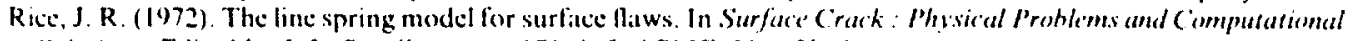
Solutions (Edited by J. L. Swedlow), pp. 171 185. ASMIE, New York.

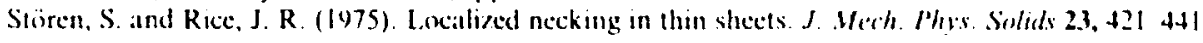

